\title{
Analysis of the Growth and Metabolites of a Pyruvate Dehydrogenase Complex- Deficient Klebsiella pneumoniae Mutant in a Glycerol-Based Medium
}

\author{
Danfeng Xu, Zongxiao Jia, Lijuan Zhang, Shuilin Fu, and Heng Gong* \\ State Key Laboratory of Bioreactor Engineering, East China University of Science and Technology, Shanghai \\ 200237, P.R. China
}

\begin{abstract}
To determine the role of pyruvate dehydrogenase complex (PDHC) in Klebsiella pneumoniae, the growth and metabolism of PDHC-deficient mutant in glycerol-based medium were analyzed and compared with those of other strains. Under aerobic conditions, the PDHC activity was fourfold higher than that of pyruvate formate lyase (PFL), and blocking of PDHC caused severe growth defect and pyruvate accumulation, indicating that the carbon flux through pyruvate to acetyl coenzyme $A$ mainly depended on PDHC. Under anaerobic conditions, although the PDHC activity was only $50 \%$ of that of PFL, blocking of PDHC resulted in more growth defect than blocking of PFL. Subsequently, combined with the requirement of $\mathrm{CO}_{2}$ and intracellular redox status, it was presumed that the critical role of PDHC was to provide NADH for the anaerobic growth of $K$. pneumoniae. This presumption was confirmed in the PDHC-deficient mutant by further blocking one of the formate dehydrogenases, FdnGHI. Besides, based on our data, it can also be suggested that an improvement in the carbon flux in the PFL-deficient mutant could be an effective strategy to construct highyielding 1,3-propanediol-producing $K$. pneumoniae strain.
\end{abstract}

Keywords: 1,3-propanediol, glycerol, Klebsiella pneumoniae, pyruvate dehydrogenase complex, pyruvate formate lyase

Received: January 24, 2018 Accepted: May 1, 2018

First published online: May 11, 2018

*Corresponding author Phone: +86-021-64253007 Fax: +86-021-64253007 E-mail: gongheng@ecust.edu.cn

pISSN 1017-7825 elSSN 1738-8872

Copyright(C) 2020 by The Korean Society for Microbiology and Biotechnology

\section{Introduction}

With growing environmental issues and limited availability of fossil fuels, much interest has been focused on the utilization of biodiesel, one of the important biofuels produced by transesterification or ethanolysis of biological feedstock [1]. However, biodiesel industry also produces glycerol of approximately $10 \mathrm{wt} . \% \mathrm{of}$ the total product [2]. Recently, owing to the rapid increase in biodiesel production, the output of glycerol has dramatically improved, decreasing the value of glycerol $[3,4]$. Therefore, it is essential to find alternative uses for glycerol. The bioconversion of glycerol into 1,3-propanediol (1,3-PD) is attractive because 1,3-PD has high value and widespread use in polyester, cosmetic, and pharmaceutical industries [5, 6]. Many microorganisms can utilize glycerol to produce 1,3-PD, and among them, Klebsiella pneumoniae is the most extensively studied with proven superior ability to produce $1,3-\mathrm{PD}[7,8]$.

The metabolic pathway of $K$. pneumoniae using glycerol as the sole carbon source is summarized in Fig. 1 . In general, glycerol is converted through reductive and oxidative pathways, with the reductive branch leading to 3hydroxypropionaldehyde formation and then 1,3-PD synthesis, while the oxidative branch providing the intermediate metabolite pyruvate and concomitantly generating the reducing power (NADH) for 1,3-PD synthesis $[9,10]$. In the reductive branch, 3-hydroxypropionaldehyde can also be oxidized to 3-hydroxypropionic acid (3-HP). Accordingly, an increase in 3-HP improved NADH generation and further promoted 1,3-PD synthesis [11]. Nevertheless, pyruvate metabolism is the most important step affecting 1,3-PD synthesis, because the metabolites originating from pyruvate lead to the decrease in carbon flux and reduction in the ability to synthesize 1,3-PD. Therefore, various genetic modifications of $K$. pneumoniae have been investigated on pyruvate node, such as blocking of the flux to lactate, acetate, or 2,3-butanediol (2,3-BD), etc. [10,12,13]. However, only few reports have focused on the key route of pyruvate to acetyl coenzyme A (AcCoA).

In Enterobacteria, pyruvate is catabolized to AcCoA by pyruvate dehydrogenase complex (PDHC) or pyruvate formate lyase (PFL), which represents a switch point between respiratory and fermentative metabolism. In general, during aerobic respiration, pyruvate is converted to $\mathrm{AcCoA}, \mathrm{CO}_{2}$, and $\mathrm{NADH}$ by PDHC, whereas during anaerobic fermentation, pyruvate is converted to AcCoA and formate by PFL [14-16]. However, evidences show that PDHC could function even under anaerobic conditions. In Escherichia coli, a significant flux through PDHC 


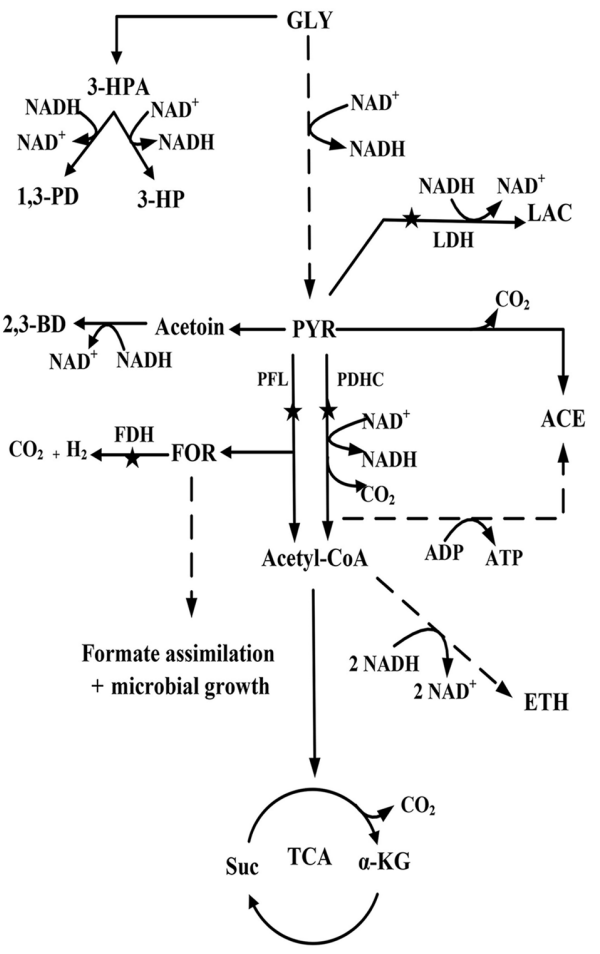

Fig. 1. Metabolic pathways of glycerol metabolism in K. pneumoniae. GLY glycerol, 3-HPA 3-hydroxypropinaldehyde, 3-HP 3-hydroxypropionic, 1,3-PD 1,3-propanediol, 2,3-BD 2,3-butanediol, PYR pyruvate, LAC lactate, FOR formate, ACE acetate, ETH ethanol, SUC succinate, $\alpha$-KG $\alpha$-ketoglutarate, TCA tricarboxylic acids cycle, PDHC pyruvate dehydrogenase complex, PFL pyruvate formate lyase, FDH formate dehydrogenase, LDH lactate dehydrogenase. Stars indicate metabolic reactions that have been blocked by gene deletions.

was observed, and the $\mathrm{CO}_{2}$ generated by PDHC was shown to be necessary for anaerobic cell growth [17]. It must be noted that 1,3-PD is a common anaerobic product during glycerol fermentation, and pyruvate catabolized by $\mathrm{PDHC}$ can generate NADH, which is beneficial for 1,3-PD production. Flux and enzyme analysis in K. pneumoniae has suggested that PDHC could function during anaerobic fermentation $[18,19]$, although the exact role of PDHC remains unclear.

In the present study, the growth and cell metabolism of PDHC-deficient mutant were analyzed in glycerolbased medium and compared with those of other mutants. It was found that blocking of PDHC resulted in severe growth defect of $K$. pneumoniae even under anaerobic condition. Furthermore, the roles of PDHC were determined, which were noted to be different from those in E. coli under anaerobic condition. Based on these data, a strategy for the construction of high-yielding 1,3-PD-producing K. pneumoniae strain was also discussed.

\section{Materials and Methods}

\section{Strains, Plasmids, and Primers}

The strains, plasmids, and primers used in this study are listed in Table 1. The lactate-deficient K. pneumoniae strain KG2 [11], derived from K. pneumoniae strain KG (CCTCC M2014574) by deleting ldhA, is a high-yielding 1,3-PD-producing strain, and was used as the parent strain in this study.

\section{Cultivation Conditions}

The parent strain was precultured in test tubes containing $5 \mathrm{ml}$ of Luria broth $(10 \mathrm{~g} / \mathrm{l}$ Difco tryptone, $5 \mathrm{~g} / \mathrm{l}$ Difco yeast extract, and $10 \mathrm{~g} / \mathrm{l} \mathrm{NaCl}$ ) for $10 \mathrm{~h}$ at $37^{\circ} \mathrm{C}$ and $200 \mathrm{rpm}$. Subsequently, for the assessment of mutants, flask batch cultures with $1 \%$ inoculum were accomplished at $37^{\circ} \mathrm{C}$ for $24 \mathrm{~h}$ with shaking at 300 and $200 \mathrm{rpm}$ under aerobic and anaerobic conditions, respectively. For aerobic cultivation, $250-\mathrm{ml}$ shake flasks containing $20 \mathrm{ml}$ of the culture medium were covered with eight layers of gauze to allow air penetration. For anaerobic cultivation, $250-\mathrm{ml}$ shake flasks containing $50 \mathrm{ml}$ of the culture medium were plugged with a gas-impermeable rubber stopper, the air in the flasks was replaced with $\mathrm{N}_{2}$ gas before cultivation, and the flasks were subsequently plugged with a gas-impermeable rubber stopper. The culture medium ( $\mathrm{pH} 7.0)$ contained $60 \mathrm{~g} / \mathrm{l}$ glycerol, $1 \mathrm{~g} / \mathrm{l}$ $\mathrm{KH}_{2} \mathrm{PO}_{4} \cdot 2 \mathrm{H}_{2} \mathrm{O}, 3.3 \mathrm{~g} / \mathrm{l}\left(\mathrm{NH}_{4}\right)_{2} \mathrm{SO}_{4}, 0.26 \mathrm{~g} / \mathrm{l} \mathrm{MgCl}_{2} \bullet 6 \mathrm{H}_{2} \mathrm{O}$, and $2 \mathrm{~g} / \mathrm{l}$ yeast extract. To investigate the effect of $\mathrm{CO}_{2}$ on bacterial growth, $1 \mathrm{~g} / 1 \mathrm{NaHCO}_{3}$ was added to the medium. 
Table 1. Strains, plasmids, primers used in this study.

\begin{tabular}{|c|c|c|}
\hline Strain, plasmid or primers & Relevant genotype and description & Reference or source \\
\hline \multicolumn{3}{|l|}{ Strains } \\
\hline K. pneumoniae KG2 & parent type, $\mathrm{Amp}^{\mathrm{r}} \Delta l d h A$ & Zhu et al. [11] \\
\hline K. pneumoniae KG-PD & $\mathrm{Amp}^{\mathrm{r}} \Delta l d h A \Delta a c e E-\mathrm{aceF}-l p d A$ & This study \\
\hline K. pneumoniae KG-PF & $\mathrm{Amp}^{\mathrm{r}} \Delta l d h A \Delta p f l A-p f l B$ & This study \\
\hline K. pneumoniae KG-PDN & $\mathrm{Amp}^{\mathrm{r}} \Delta l d h A \Delta a c e E-\mathrm{aceF}-l p d A \Delta f d n G-f d n H-f d n I$ & This study \\
\hline \multicolumn{3}{|l|}{ Plasmids } \\
\hline pKD4 & $\mathrm{Kan}^{\mathrm{r}}, \mathrm{Amp}^{\mathrm{r}}$, ori R6Kgamma, rgnB & This lab \\
\hline pKD46-Tc & $\mathrm{Tc}^{\mathrm{r}}$, repA101(ts),oriR101, araBp-gam-bet-exo & This lab \\
\hline $\mathrm{pCP} 20-\mathrm{Tc}$ & $\mathrm{Tc}^{\mathrm{r}}, \mathrm{Cm}^{\mathrm{r}}$, ts-rep, [cI857] (lambda)(ts), FLP & This lab \\
\hline pMD 18T & Cloning vector, $\mathrm{Amp}^{\mathrm{r}}$ & TAKARA \\
\hline \multicolumn{3}{|l|}{ Primers } \\
\hline aceE-1 & 5' GAAATGCTCCCGTTGGTC 3' & \\
\hline aceE-2 & $\begin{array}{l}\text { 5' AAGCAGCTCCAGCCTACACATTAA } \\
\text { GAACCAGAGTTCTCGT 3' }\end{array}$ & \\
\hline $\operatorname{lpdA-3}$ & $\begin{array}{l}\text { 5' AGGAGGATATTCATATGGACGGCG } \\
\text { CGATTGTCGGCACC 3' }\end{array}$ & \\
\hline $\operatorname{lpdA-4}$ & 5' TTACTTTTTCTTCGCTTTGG 3' & \\
\hline pflA-1 & 5' ACTTTGTGACCATACT 3' & \\
\hline pflA-2 & $\begin{array}{l}\text { 5' AAGCAGCTCCAGCCTACACAGATG } \\
\text { ATTCCGCGCACC } 3 \text { ' }\end{array}$ & \\
\hline pflB-3 & $\begin{array}{l}\text { 5' AGGAGGATATTCATATGGACGCAG } \\
\text { TGTCAAAATCAACAGG 3' }\end{array}$ & \\
\hline$p f l B-4$ & 5' GCCTGGGAAGGTTTTG 3' & \\
\hline$f d n G-1$ & 5' CACTGGGTGGATATCA 3' & \\
\hline$f d n G-2$ & $\begin{array}{l}\text { 5' AAGCAGCTCCAGCCTACACAGTAC } \\
\text { CGGAACGAATCG 3' }\end{array}$ & \\
\hline$f d n I-3$ & $\begin{array}{l}\text { 5' AGGAGGATATTCATATGGACGCCT } \\
\text { GCTGATTCATGC 3' }\end{array}$ & \\
\hline$f d n I-4$ & 5' CCTTCACGACTCTCTTTCAT 3' & \\
\hline
\end{tabular}

\section{Construction of PDHC-Deficient Mutant and Other Mutants}

The fragment containing $a c e E, a c e F$, and $l p d A$ was deleted for construction of PDHC-deficient mutant. In addition, the fragment containing $p f l A$ and $p f l B$ was deleted for construction of $p f l A-p f l B$ mutant, and the fragment containing $f d n G, f d n H$, and $f d n I$ was deleted for construction of $f d n G-f d n H-f d n I$ mutant. All the mutants were derived from the parent strain K. pneumoniae KG2 by using lambda Red recombination [20]. The fragment disruption cassettes with a kanamycin resistance marker and flippase recognition site were amplified by two-step PCR from the chromosomal DNA of K. pneumoniae KG2 and pKD4 vector, respectively, using the primers listed in Table 1. For the construction of PDHC-deficient mutant, pKD46-Tc was first transformed into K. pneumoniae KG2, followed by transformation with the fragment-PD disruption cassette. The recombinant strain was selected from Luria-Bertani (LB) plates supplemented with kanamycin after a 24 -h culture at $37^{\circ} \mathrm{C}$. Then, pCP20-Tc was transformed into the recombinant strain and incubated at $37^{\circ} \mathrm{C}$ to remove the kanamycin resistance gene. Finally, the transformants were diluted and plated onto solid LB medium at $42^{\circ} \mathrm{C}$ to obtain a PDHC-deficient mutant named KG-PD that was no longer resistant to kanamycin and tetracycline hydrochloride. Similarly, by using the same procedure, $p f l A-p f l B$ was deleted from the genome of $K$. pneumoniae KG2 to obtain the mutant KG-PF. The mutant KG-PDN was derived from KG-PD by further deletion of $f d n G-f d n H-f d n I$. All the mutants derived in this study are listed in Table 1.

\section{Metabolites Analysis}

The optical density (OD) of the bacterial cultures was related to the dry cell weight by an experimentally determined calibration curve. The OD of the bacterial cultures at $620 \mathrm{~nm}\left(\mathrm{OD}_{620}\right)$ was measured after appropriate dilution. The concentrations of 1,3-PD, 2,3-BD, ethanol, and acetoin in the fermentation broths were quantified by using gas chromatography. The analysis conditions included $\mathrm{N}_{2}$ as the carrier gas, detector temperature of $270^{\circ} \mathrm{C}$, and column temperature of $110^{\circ} \mathrm{C}$. Other metabolites present in the fermentation broths were quantified using high-performance liquid chromatography system equipped with a 2487 Dual-Wavelength Absorbance Detector (Waters Corporation, USA) and a Plastisol ODS column (AQ-C18, $5 \mu \mathrm{m}, 250 \times 4.6 \mathrm{~mm}$; Welch Material, Inc., USA) at a flow rate of $0.6 \mathrm{ml} / \mathrm{min}$ and column temperature of $60^{\circ} \mathrm{C}$. The mobile phase was $2.5 \mathrm{mM} \mathrm{H}_{2} \mathrm{SO}_{4}$. For the investigation of extracellular metabolites, $1 \mathrm{ml}$ of the culture was centrifuged for $1 \mathrm{~min}$ at $12,000 \times \mathrm{g}$ in a microcentrifuge and the supernatant was filtered through a $0.45-\mu \mathrm{m}$ syringe filter before analysis.

\section{Enzyme Assays and Determination of $\mathrm{NAD}^{+}$and NADH}

The activities of PDHC and PFL were assayed using the methods described previously [21,22]. Crude protein was measured by the method of Bradford with bovine serum albumin as the standard [23]. For anaerobic enzyme assays, strict anoxic condition was employed as described previously [24]. The cells were harvested after $12 \mathrm{~h}$ of 

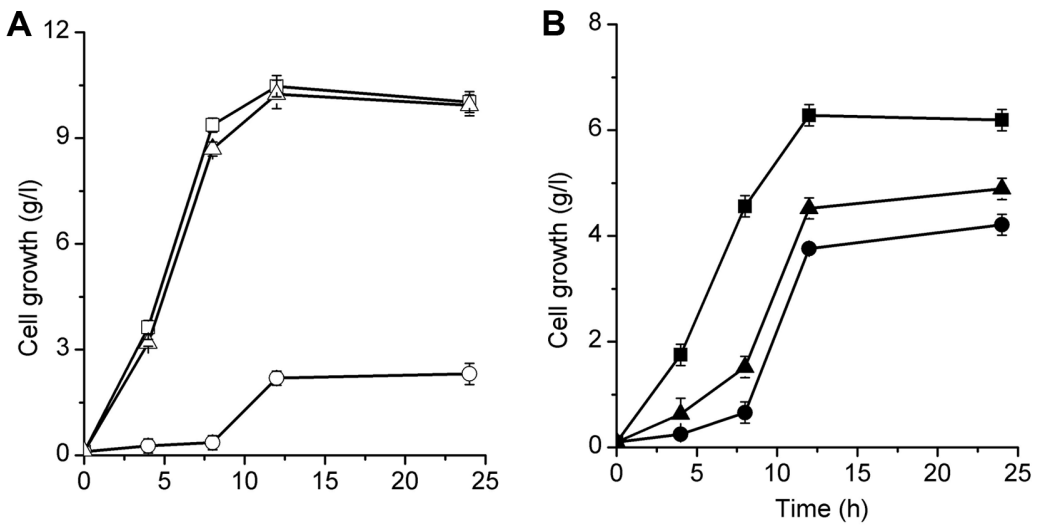

Fig. 2. Cell growth of KG2 (square), KG-PD (circle) and KG-PF (triangle) during a $24 \mathrm{~h}$ batch flask cultivation under aerobic (empty symbol) and anaerobic (solid symbol) conditions. Date points are average of three identical experiments.

flask culture, and then washed thrice with cold $50 \mathrm{mM}$ potassium phosphate buffer containing $1 \mathrm{mM}$ dithiothreitol ( $\mathrm{pH}$ 7.0). The resuspended cells were disrupted by freezing and thawing, and the supernatant was harvested after centrifugation for $20 \mathrm{~min}$ at $12,000 \times g$ at $4^{\circ} \mathrm{C}$ for enzyme detection. One unit (U) of enzyme activity represents the amount of enzyme catalyzing the conversion of $1 \mu \mathrm{mol}$ of substrate per min into specific products, and $\mathrm{U} / \mathrm{mg}$ refers to the enzyme activities in $1 \mathrm{mg}$ of crude protein extract. The intracellular concentrations of $\mathrm{NAD}^{+}$and $\mathrm{NADH}$ were determined using the procedures described by Cui et al. [12].

\section{Results}

\section{Growth of the PDHC-Deficient Mutant}

PDHC contains three enzymatic components: E1, E2, and E3, which are encoded by aceE, aceF, and $l p d A$, respectively [25]. In the present study, these three contiguous genes were deleted for the construction of the PDHC-deficient mutant, KG-PD. The growth of KG-PD during batch culture in glycerol-based medium is shown in Fig. 2. As aerobic catabolism of pyruvate to AcCoA has been reported to be mainly dependent on PDHC [26], as expected, the aerobic growth of KG-PD was severely inhibited, with cell concentration decreasing to $2.31 \mathrm{~g} / \mathrm{l}$, which was only $23 \%$ of that of the parent strain (Fig. 2A) at the end of the culture period. Interestingly, KG-PD exhibited growth defect under anaerobic conditions as well, with the cell concentration decreasing to $4.21 \mathrm{~g} / \mathrm{l}$ after 24 h of culture, which was $68 \%$ of that of K. pneumoniae KG2 (Fig. 2B).

It has been reported that under anaerobic condition, the catabolism of pyruvate to AcCoA is mainly dependent on PFL [27]. The growth of the pflA-pflB mutant, KG-PF, is shown in Fig. 2. As expected, the aerobic growth of KG$\mathrm{PF}$ was almost the same as that of K. pneumoniae KG2, whereas a growth defect of KG-PF was observed under anaerobic condition. However, unexpectedly, the anaerobic growth of KG-PF was better than that of KG-PD. Thus, these findings strongly indicated that $\mathrm{PDHC}$ was not only required for aerobic but also for anaerobic catabolism of pyruvate to AcCoA in K. pneumoniae.

\section{Analysis of the Metabolites and Enzymes of the PDHC-Deficient Mutant}

The metabolites of the PDHC-deficient mutant, KG-PD, determined after $24 \mathrm{~h}$ of batch culture are listed in Table 2. As the parent stain is a lactate-deficient 1,3-PD producer, the 1,3-PD level reached 10.2 and $18.5 \mathrm{~g} / \mathrm{l}$ at the end of aerobic and anaerobic culture, respectively. While formate was almost undetected, the other three byproducts originating from pyruvate could be detected: 2,3-BD was the main byproduct and its accumulation was higher during aerobic culture $(6.12 \mathrm{~g} / \mathrm{l})$, whereas the accumulation of acetate and ethanol was higher during anaerobic culture (1.44 and $3.52 \mathrm{~g} / \mathrm{l}$, respectively, at the end of batch culture with K. pneumoniae KG2). Furthermore, high concentration of acetoin, the precursor of 2,3-BD, was noted (2.12 g/l) in K. pneumoniae KG2 under aerobic condition.

When compared with K. pneumoniae KG2, the PDHC-deficient mutant, KG-PD, exhibited high accumulation of pyruvate $(3.98 \mathrm{~g} / \mathrm{l})$ under aerobic condition. However, under anaerobic condition, no accumulation of pyruvate in KG-PD was noted, when compared with that in K. pneumoniae KG2. With regard to the other metabolites $(1,3-$ $\mathrm{PD}, 2,3-\mathrm{BD}$, and ethanol), their accumulation was extremely low in KG-PD under both aerobic and anaerobic conditions, while the accumulation of acetate slightly increased during anaerobic cultivation. Table 2 presents the accumulation of metabolites in the pflA-pflB mutant, KG-PF, after $24 \mathrm{~h}$ of flask culture. It can be noted from the table that under both aerobic and anaerobic conditions, pyruvate was not accumulated, whereas the accumulation of 1,3-PD and 2,3-BD increased and that of ethanol decreased.

The activities of PDHC and PFL in different strains after $12 \mathrm{~h}$ of flask culture are shown in Fig. 3. Under aerobic condition, the PDHC activity in the parent strain K. pneumoniae KG2 was $1.20 \mathrm{U} / \mathrm{mg}$, which was nearly fourfold higher than that of PFL (Fig. 3A). However, in KG-PD, the activity of PDHC was completely eliminated, combined with severe growth defect and high accumulation of pyruvate, clearly suggesting that PDHC is responsible for the 
Table 2. Metabolites of KG2, KG-PD, KG-PF, KG-PDN in glycerol-based medium under aerobic and anaerobic conditions at $24 \mathrm{~h}$ in shake flask.

\begin{tabular}{|c|c|c|c|c|c|c|c|c|}
\hline & \multicolumn{4}{|c|}{ Aerobic } & \multicolumn{4}{|c|}{ Anaerobic } \\
\hline & KG2 & KG-PD & KG-PF & KG-PDN & KG2 & KG-PD & KG-PF & KG-PDN \\
\hline $1,3-\mathrm{PD}(\mathrm{g} / \mathrm{l})$ & $10.2 \pm 0.4$ & $2.1 \pm 0.3$ & $13.0 \pm 0.3$ & $10.4 \pm 0.4$ & $18.5 \pm 0.4$ & $10.5 \pm 0.3$ & $20.3 \pm 0.3$ & $10.2 \pm 0.4$ \\
\hline $2,3-\mathrm{BD}(\mathrm{g} / \mathrm{l})$ & $6.12 \pm 0.31$ & $\mathrm{ND}^{\mathrm{a}}$ & $7.22 \pm 0.22$ & $2.62 \pm 0.31$ & $4.71 \pm 0.30$ & $2.32 \pm 0.11$ & $6.42 \pm 0.12$ & $4.11 \pm 0.21$ \\
\hline Acetoin (g/l) & $2.11 \pm 0.20$ & ND & $3.00 \pm 0.20$ & $9.51 \pm 0.20$ & ND & ND & ND & ND \\
\hline Ethanol (g/l) & $2.32 \pm 0.31$ & ND & ND & ND & $3.52 \pm 0.31$ & $1.51 \pm 0.22$ & $0.61 \pm 0.23$ & ND \\
\hline Pyruvate (g/l) & $0.04 \pm 0.02$ & $3.98 \pm 0.02$ & $0.05 \pm 0.01$ & $0.04 \pm 0.02$ & $0.06 \pm 0.01$ & $0.04 \pm 0.02$ & $0.06 \pm 0.01$ & $0.05 \pm 0.02$ \\
\hline Formate (g/l) & $0.06 \pm 0.02$ & $0.08 \pm 0.02$ & ND & $0.07 \pm 0.02$ & $0.08 \pm 0.02$ & $0.06 \pm 0.02$ & ND & $0.06 \pm 0.02$ \\
\hline 3-HP (g/l) & $0.35 \pm 0.05$ & $0.40 \pm 0.02$ & $0.21 \pm 0.02$ & $3.57 \pm 0.03$ & $0.23 \pm 0.03$ & $0.45 \pm 0.02$ & $0.18 \pm 0.03$ & $0.35 \pm 0.03$ \\
\hline Acetate $(\mathrm{g} / \mathrm{l})$ & $0.53 \pm 0.02$ & ND & $0.48 \pm 0.05$ & $0.50 \pm 0.04$ & $1.44 \pm 0.03$ & $1.72 \pm 0.03$ & $1.20 \pm 0.02$ & $2.34 \pm 0.02$ \\
\hline $\begin{array}{l}\text { Glycerol } \\
\text { consumption } \\
(\mathrm{g} / \mathrm{l})\end{array}$ & $53.2 \pm 0.4$ & $22.9 \pm 0.5$ & $57.2 \pm 0.4$ & $53.9 \pm 0.6$ & $49.7 \pm 0.5$ & $40.4 \pm 0.4$ & $50.8 \pm 0.5$ & $38.6 \pm 0.8$ \\
\hline
\end{tabular}

All results are expressed as the mean \pm SD of three independent experiments.

${ }^{\mathrm{a}} \mathrm{ND}$, not detected.

generation of carbon flux to AcCoA needed for cell growth under aerobic condition. During anaerobic cultivation, the carbon flux to AcCoA in K. pneumoniae KG2 appeared to mainly depend on PFL, with its activity reaching $0.81 \mathrm{U} / \mathrm{mg}$, which was nearly twofold higher than that of PDHC. Accordingly, in KG-PF, extremely low PFL activity (Fig. 3B) was accompanied by severe anaerobic growth defect caused by the lack of carbon flux to AcCoA. However, more severe anaerobic growth defect (Fig. 2B) was noted in KG-PD. Thus, the role of PDHC in anaerobic cultivation of K. pneumonia may not be limited to the generation of carbon flux to AcCoA.

When compared with PFL, pyruvate catalyzed by PDHC generates NADH, which could affect the redox status of the cells. The redox status of strains was analyzed. The ratios of $\mathrm{NADH} / \mathrm{NAD}^{+}$in mutants under aerobic condition maintained the same level as that in the parent strain KG2 (data no shown), and the variations in $\mathrm{NADH} / \mathrm{NAD}^{+}$in strains under anaerobic condition are shown in Fig. 4. In the parent strain K. pneumoniae KG2 and the pflA-pflB mutant, KG-PF, the NADH:NAD ${ }^{+}$ratios were around $0.20-0.25$ and about 0.25 at the end of anaerobic culture. However, in the PDHC-deficient strain, KG-PD, the redox balance was disrupted, with the $\mathrm{NADH}: \mathrm{NAD}^{+}$ratio decreasing to 0.09 at the end of the anaerobic culture, which was only $35 \%$ of that in $K$. pneumoniae KG2.

\section{Further Deletion of $f d n G-f d n H-f d n I$ in the PDHC-Deficient Mutant}

Besides generating carbon flux to $\mathrm{AcCoA}$ and NADH, pyruvate catabolized by $\mathrm{PDHC}$ also generates $\mathrm{CO}_{2}$. It has been reported that the generation of $\mathrm{CO}_{2}$ by $\mathrm{PDHC}$ is required for the anaerobic growth of E. coli [17]. To investigate the effect of $\mathrm{CO}_{2}$ on the anaerobic growth of KG-PD, $1 \mathrm{~g} / \mathrm{NaHCO}_{3}$, an efficient source of $\mathrm{CO}_{2}$ for bacterial growth $[28,29]$, was added to the culture medium. As shown in Fig. 5, anaerobic growth was not promoted by the addition of $\mathrm{NaHCO}_{3}$. It has been demonstrated that the conversion of pyruvate to acetate catalyzed by pyruvate oxidase (PoxB) in $\mathrm{K}$. pneumoniae can generate $\mathrm{CO}_{2}$, which can effectively provide endogenous $\mathrm{CO}_{2}$ for anaerobic growth [10]. In the present study, we found that more acetate was produced in KGPD during anaerobic cultivation (Table 2).

As the reaction of formate catalyzed by formate dehydrogenase (FDH) is also an effective mode of endogenous $\mathrm{CO}_{2}$ generation [30], the effect of blocking of FDH on the PDHC-deficient mutant was further analyzed. Three
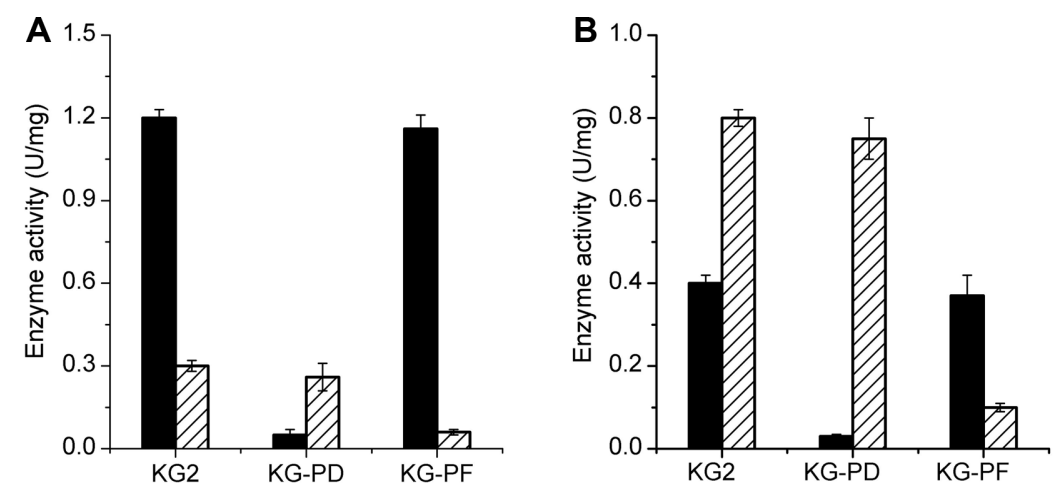

Fig. 3. The pyruvate dehydrogenase complex and pyruvate formate lyase activities of parent strain KG2 and mutant strains of KG-PD and KG-PF under aerobic condition (a) and anaerobic condition (b) after $12 \mathrm{~h}$ batch flask cultivation. Black bars represent PDHC activity while hatched bars represent PFL activity. Error bars represent the standard deviations from three independent experiments. 


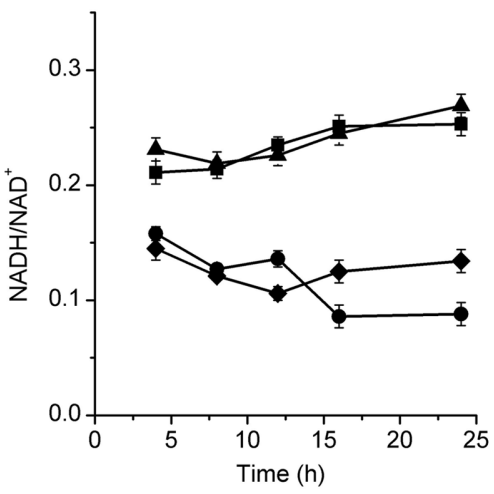

Fig. 4. Intracellular redox balance (NADH/NAD ${ }^{+}$) profiles during anaerobic flask culture of KG2 (square), KG-PD (circle), KG-PF (triangle), and KG-PDN (rhombus). Date points are averages of at least three identic experiments.

kinds of FDHs have been reported, namely, FdnGHI (FDH-N) induced by anaerobiosis and nitrate, FdoGHI (FDH-O) synthesized in the presence of oxygen, and FdhF (FDH-H) synthesized at optimal level during fermentative growth of E. coli $[31,32]$. All these three FDHs were respectively blocked in the PDHC-deficient mutant; however, only blocking of FdnGHI, encoded by $f d n G-f d n H-f d n I$, showed effects on the PDHC-deficient mutant under our experimental conditions (data not shown). Under anaerobic conditions, blocking of FdnGHI resulted in further growth defect of the mutant KG-PDN. When compared with KG-PD, the cell concentration of KG-PDN decreased from 3.89 to $3.21 \mathrm{~g} / \mathrm{l}$ (Fig. 5) at the end of anaerobic cultivation, and the production of acetate increased and reached $2.34 \mathrm{~g} / \mathrm{l}$ (Table 2). However, when the culture medium was supplied with $\mathrm{NaHCO}_{3}$, the anaerobic growth of KG-PDN recovered and reached the same level as that of KG-PD (Fig. 5). Figure 4 also shows the redox status in KG-PDN. A further blocking of FdnGHI appeared to result in better redox status, and at the later stage (after $15 \mathrm{~h}$ ) of anaerobic growth of KG-PDN, the NADH/NAD+ ratio remained at around 0.15 , which was higher than that in KG-PD (around 0.09). Thus, these findings effectively confirmed the presence of sufficient internal $\mathrm{CO}_{2}$ pool to meet the anaerobic growth of the PDHC-deficient mutant, KG-PD.

Interestingly, the severe aerobic growth defect noted in KG-PD was completely recovered by further blocking of FdnGHI, and at the end of flask culture under aerobic condition, the cell growth of KG-PDN reached $9.93 \mathrm{~g} / \mathrm{l}$, which was almost the same as that of the parent strain. Besides, excessive accumulation of pyruvate disappeared, and when compared with those in the parent strain KG2, high concentrations of acetoin and 3-HP were formed in KG-PDN during aerobic cultivation, which reached 9.5 and $3.57 \mathrm{~g} / \mathrm{l}$, respectively, at the end of cultivation (Table 2).

\section{Discussion}

For bioconversion of glycerol to 1,3-PD by K. pneumoniae, cell metabolism in pyruvate node should be carefully controlled [33]. The PDHC occupies the key position in the oxidation of pyruvate to AcCoA. However, to date, only few studies have focused on the role of PDHC in K. pneumoniae, especially under anaerobic conditions. In the present study, the cell growth and metabolites of PDHC-deficient mutant were analyzed and compared with other strains, and the role of PDHC in K. pneumoniae was examined.

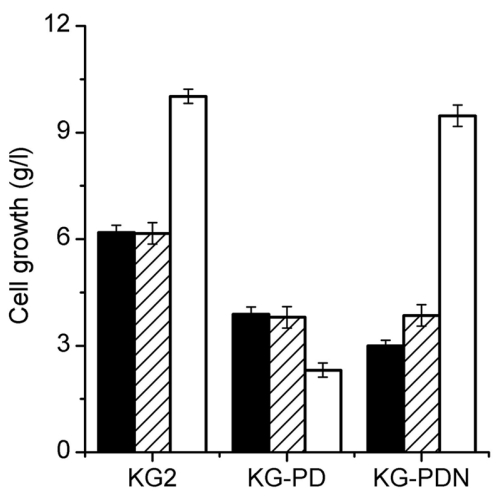

Fig. 5. The cell growth of KG2, KG-PD and KG-PDN at the end of flask cultivation. Black bars without $\mathrm{NaHCO}_{3}$, hatched bars with $\mathrm{NaHCO}_{3}$, both were cultured under anaerobic condition. Empty bars represent cell growth without $\mathrm{NaHCO}_{3}$ under aerobic condition. Error bars represent the standard deviations from three independent experiments. 
Under aerobic condition, pyruvate is mainly converted to AcCoA by PDHC in K. pneumoniae, and blocking of PDHC results in high accumulation of pyruvate and severe growth defect. Although PFL has been reported to function during aerobic cultivation of E. coli [34], the data of the present study indicated that the carbon flux to AcCoA required for aerobic growth of K. pneumonia seemed to be completely used by PDHC alone, and to balance the extra NADH, the pflA-pflB mutant secreted more 1,3-PD and 2,3-BD. However, interestingly, the level of ethanol in the $p f l A-p f l B$ mutant severely decreased to undetectable level, which should in fact increase because conversion of one molecule of AcCoA to ethanol needs two molecules of $\mathrm{NADH}$, which might be more favorable for the cells to balance the extra $\mathrm{NADH}$, when compared with secretion of 1,3-PD or 2,3-BD [35]. The probable reason for this observation could be that all of the AcCoA molecules in the pflA-pflB mutant were used for cell growth, resulting in no extra AcCoA for ethanol formation. Thus, in addition to the function of maintaining the cell redox status [36], ethanol seems to be an indicator of AcCoA pool required for cell growth in K. pneumoniae.

Under anaerobic conditions, pyruvate is mainly converted to AcCoA by PFL. However, blocking of PDHC results in more severe growth inhibition, when compared with that of PFL, indicating that the role of PDHC may not only be limited to the generation of carbon flux to AcCoA during anaerobic cultivation of K. pneumoniae. It has been reported that the critical role of PDHC is to generate $\mathrm{CO}_{2}$ needed for the anaerobic growth of $E$. coli. However, in the present study, addition of extra $\mathrm{CO}_{2}$ did not promote cell growth and caused an imbalance in the redox status in the PDHC-deficient mutant, thus strongly indicating that the critical role of PDHC is generation of $\mathrm{NADH}$ and not $\mathrm{CO}_{2}$ for anaerobic growth of $K$. pneumoniae. Further studies are needed to explore the reason for the anaerobic growth of $K$. pneumoniae using excess NADH, which could be an important feature explaining the ability of $K$. pneumoniae to produce high amount of 1,3-PD. The findings of the present study also showed that $K$. pneumoniae can balance the extra NADH to produce more 1,3-PD by blocking PFL during anaerobic cultivation. Moreover, despite the anaerobic growth defect, highest 1,3-PD production was noted in the pflA-pflB mutant. In previous studies on $E$. coli and K. pneumoniae, anaerobic growth defects of $p f l B$ mutants have been reported, which were presumed to be owing to the redox imbalance caused by ineffective regeneration of $\mathrm{NAD}^{+}$ $[34,37,38]$. However, in the present study, combined with the balanced redox status and low level of ethanol, the lack of AcCoA pool could be the reason for the anaerobic growth defect of the $p f l A-p f l B$ mutant. Thus, an improvement in the carbon flux for fixing the growth defect of the $p f l A-p f l B$ mutant could be an effective strategy for constructing the high-yielding 1,3-PD-producing K. pneumoniae strain.

Unlike PDHC, there could be several PFLs in the cells, and it has been reported that PFL-I (encoded by $p f l B$ and $p f l A$ ) is dominant in E. coli [39]. Similarly, in the present study, deletion of $p f l A-p f l B$ severely blocked the activities of PFL in K. pneumoniae. PFL catalyzes the conversion of pyruvate to AcCoA and formate. Formate is an important electron donor, which is oxidized by $\mathrm{FDH}$ to generate $\mathrm{CO}_{2}$. In the PDHC-deficient mutant, almost no accumulation of formate was noted, suggesting high activity of FDH in K. pneumoniae. Therefore, the effect of blocking of FDH on the PDHC-deficient mutant was further analyzed. Among the three kinds of FDHs blocked, only blocking of FdnGHI showed the effects on PDHC-deficient K. pneumoniae under our experimental conditions.

Under anaerobic conditions, further blocking of FdnGHI in the PDHC-deficient mutant resulted in a decrease in cell growth, which was recovered by the addition of $\mathrm{CO}_{2}$, indicating the lack of $\mathrm{CO}_{2}$ in the FdnGHI/PDHC double-deficient mutant. Furthermore, sufficient $\mathrm{CO}_{2}$ pool for anaerobic growth in the PDHC-deficient mutant was confirmed. Thus, combined with the data of acetate production and redox status, it can be concluded that: 1) blocking of PDHC enhances the PoxB pathway in the cells to maintain the $\mathrm{CO}_{2}$ level for anaerobic growth of K. pneumoniae, and the growth defect in this case was caused by the imbalance of redox status; and 2) blocking of both PDHC and FdnGHI resulted in lack of $\mathrm{CO}_{2}$ being the key inhibiting factor and further decrease in anaerobic growth, and a better level of redox status was noted in this case, when compared with that found following blocking of PDHC only.

Interestingly, under aerobic conditions, an unexpected good growth of FdnGHI/PDHC double-deficient mutant was observed. Nevertheless, further studies are required to determine how blocking of FdnGHI promotes aerobic growth of PDHC-deficient mutant. As formate, which is also a carbon source [40], was not accumulated following blocking of FdnGHI, the cells seemed to reutilize formate to provide carbon flux, which might have been acquired from pyruvate catabolized by PDHC, for cell growth in FdnGHI/PDHC double-deficient mutant. Nevertheless, blocking of FdnGHI and PDHC triggered metabolic flux redistribution during aerobic cultivation of K. pneumoniae, resulting in high production of 3-HP and acetoin, which could probably be attributed to the lack of PDHC $[11,13]$. It should be noted that the FdnGHI/PDHC double-deficient mutant showed the ability for the production acetoin, which is also a valuable platform chemical in recent times [41, 42].

In summary, the role of PDHC in K. pneumoniae was analyzed in glycerol-based medium. As expected, under aerobic condition, the carbon flux from pyruvate to AcCoA mainly depended on PDHC, whereas under anaerobic condition, the critical role of PDHC appeared to provide NADH for the balance of redox status. Based on our findings, it can be suggested that an improvement in the carbon flux in the PFL-deficient mutant might be an efficient strategy to construct a high-yielding 1,3-PD-producing K. pneumoniae, and that FdnGHI/PDHC double-deficient mutant has the ability to produce acetoin.

\section{Acknowledgment}

This work was supported by the National Natural Science Foundation of China under Grant No. 31271862. 


\section{Conflict Interest}

The authors have no financial conflicts of interest to declare.

\section{References}

1. Teng WK, Ngoh GC, Yusoff R, Aroua MK. 2016. Microwave-assisted transesterification of industrial grade crude glycerol for the production of glycerol carbonate. Chem. Eng. J. 284: 469-477.

2. Dobson R, Gray V, Rumbold K. 2012. Microbial utilization of crude glycerol for the production of value-added products. J. Ind. Microbiol. Biotechnol 39: 217-226.

3. Quispe CAG, Coronado CJR, Carvalho JA. 2013. Glycerol: Production, consumption, prices, characterization and new trends in combustion. Renew. Sustain. Energy Rev. 27: 475-493.

4. Xiu ZL, Zeng AP. 2008. Present state and perspective of downstream processing of biologically produced 1,3-propanediol and 2,3butanediol. Appl. Microbiol. Biotechnol. 78: 917-926.

5. Xu G, Liu Y, Gao Q. 2016. Multi-objective optimization of a continuous bio-dissimilation process of glycerol to 1, 3-propanediol. J. Biotechnol. 219: 59-71.

6. Zhang YM, Luo JA, Zhao XB, Liu DH. 2015. A novel strategy for 1,3-propanediol recovery from fermentation broth and control of product colority using scraped thin-film evaporation for desalination. RSC Adv. 5: 48269-48274.

7. Gungormusler-Yilmaz M, Cicek N, Levin DB, Azbar N. 2016. Cell immobilization for microbial production of 1,3-propanediol. Crit. Rev. Biotechnol. 36: 482-494.

8. Gao LR, Jiang X, Fu SL, Gong H. 2014. In silico identification of potential virulence genes in 1,3-propanediol producer Klebsiella pneumonia. J. Biotechnol. 189: 9-14.

9. Huang YN, Li ZM, Shimizu K, Ye Q. 2012. Simultaneous production of 3-hydroxypropionic acid and 1,3-propanediol from glycerol by a recombinant strain of Klebsiella pneumoniae. Bioresour. Technol. 103: 351-359.

10. Lin J, Zhang YQ, Xu DF, Xiang G, Jia ZX, Fu SL, et al. 2016. Deletion of poxB, pta, and ackA improves 1,3-propanediol production by Klebsiella pneumoniae. Appl. Microbiol. Biotechnol. 100: 2775-2784.

11. Zhu CQ, Jiang X, Zhang YQ, Lin J, Fu SL, Gong H. 2015. Improvement of 1,3-propanediol production in Klebsiella pneumoniae by moderate expression of puuC (encoding an aldehyde dehydrogenase). Biotechnol. Lett. 37: 1783-1790.

12. Cui YL, Zhou JJ, Gao LR, Zhu CQ, Jiang X, Fu SL, et al. 2014. Utilization of excess NADH in 2,3-butanediol-deficient Klebsiella pneumoniae for 1,3-propanediol production. J. Appl. Microbiol. 117: 690-698.

13. Xu YZ, Guo NN, Zheng ZM, Ou XJ, Liu HJ, Liu DH. 2009. Metabolism in 1,3-Propanediol Fed-Batch Fermentation by a D-Lactate Deficient Mutant of Klebsiella pneumoniae. Biotechnol. Bioeng. 104: 965-972.

14. Snoep JL, de Graef MR, Westphal AH, de Kok A, Teixeira de Mattos MJ, Neijssel OM. 1993. Differences in sensitivity to NADH of purified pyruvate dehydrogenase complexes of Enterococcus faecalis, Lactococcus lactis, Azotobacter vinelandii and Escherichia coli: implications for their activity in vivo. FEMS Microbiol. Lett. 114: 279-283.

15. Wang QZ, Ou MS, Kim Y, Ingram LO, Shanmugam KT. 2010. Metabolic Flux Control at the Pyruvate Node in an Anaerobic Escherichia coli Strain with an Active Pyruvate Dehydrogenase. Appl. Environ. Microbiol. 76: 2107-2114.

16. Wilkinson KD, Williams CH, Jr. 1981. NADH inhibition and NAD activation of Escherichia coli lipoamide dehydrogenase catalyzing the NADH-lipoamide reaction. J. Biol. Chem. 256: 2307-2314.

17. Murarka A, Clomburg JM, Moran S, Shanks JV, Gonzalez R. 2010. Metabolic analysis of wild-type Escherichia coli and a pyruvate dehydrogenase complex (PDHC)-deficient derivative reveals the role of PDHC in the fermentative metabolism of glucose. J. Biol. Chem. 285: 31548-31558.

18. Menzel K, Ahrens K, Zeng A, Deckwer W. 1998. Kinetic, dynamic, and pathway studies of glycerol metabolism by Klebsiella pneumoniae in anaerobic continuous culture: IV. Enzymes and fluxes of pyruvate metabolism. Biotechnol. Bioeng. 60: 617-626.

19. Menzel K, Zeng AP, Deckwer WD. 1997. Enzymatic evidence for an involvement of pyruvate dehydrogenase in the anaerobic glycerol metabolism of Klebsiella pneumoniae. J. Biotechnol. 56: 135-142.

20. Yamamoto S, Izumiya H, Morita M, Arakawa E, Watanabe H. 2009. Application of lambda red recombination system to Vibrio cholerae genetics: simple methods for inactivation and modification of chromosomal genes. Gene 438: 57-64.

21. Garrigues C, Loubiere P, Lindley ND, Cocaign-Bousquet M. 1997. Control of the shift from homolactic acid to mixed-acid fermentation in Lactococcus lactis: predominant role of the NADH/NAD ${ }^{+}$ratio. J. Bacteriol. 179: 5282-5287.

22. Russell GC, Guest JR. 1990. Overexpression of restructured pyruvate dehydrogenase complexes and site-directed mutagenesis of a potential active-site histidine residue. Biochem. J. 269: 443-450.

23. Bradford MM. 1976. A rapid and sensitive method for the quantitation of microgram quantities of protein utilizing the principle of protein-dye binding. Anal. Biochem. 72: 248-254.

24. Samuelov NS, Lamed R, Lowe S, Zeikus JG. 1991. Influence of $\mathrm{CO}_{2}-\mathrm{HCO}_{3}$ levels and $\mathrm{pH}$ on growth, succinate production, and enzyme activities of Anaerobiospirillum succiniciproducens. Appl. Environ. Microbiol. 57: 3013-3019.

25. Stephens PE, Darlison MG, Lewis HM, Guest JR. 1983. The pyruvate dehydrogenase complex of Escherichia coli K12. Nucleotide sequence encoding the dihydrolipoamide acetyltransferase component. Eur. J. Biochem. 133: 481-489.

26. Eikmanns BJ, Blombach B. 2014. The pyruvate dehydrogenase complex of Corynebacterium glutamicum: an attractive target for metabolic engineering. J. Biotechnol. 192 Pt B: 339-345.

27. Skorokhodova AY, Morzhakova AA, Gulevich AY, Debabov VG. 2015. Manipulating pyruvate to acetyl-CoA conversion in Escherichia coli for anaerobic succinate biosynthesis from glucose with the yield close to the stoichiometric maximum. J. Biotechnol. 214: $33-42$.

28. Alscher G, Krug H, Liebig HP. 2001. Optimisation of $\mathrm{CO}_{2}$ and temperature control in greenhouse crops by means of growth models at different abstraction levels - I. Control strategies, growth models and input data. Gartenbauwissenschaft 66: 105-114.

29. Hsu HH, Abbo BG. 2004. Role of bicarbonate/CO ${ }^{2}$ buffer in the initiation of vesicle-mediated calcification: mechanisms of aortic calcification related to atherosclerosis. Biochim. Biophys. Acta 1690: 118-123.

30. Sawers RG, Blokesch M, Bock A. 2004. Anaerobic formate and hydrogen metabolism. EcoSal Plus. 1(1). doi: 10.1128/ecosalplus.3.5.4.

31. Beyer L, Doberenz C, Falke D, Hunger D, Suppmann B, Sawers RG. 2013. Coordination of FocA and pyruvate formate-lyase synthesis in Escherichia coli demonstrates preferential translocation of formate over other mixed-acid fermentation products. J. Bacteriol. 195: 1428-1435.

32. Thomé R, Gust A, Toci R, Mendel R, Bittner F, Magalon A, et al. 2012. A sulfurtransferase is essential for activity of formate dehydrogenases in Escherichia coli. J. Biol. Chem. 287: 4671-4678.

33. Wu Z, Wang Z, Wang G, Tan T. 2013. Improved 1,3-propanediol production by engineering the 2,3-butanediol and formic acid pathways in integrative recombinant Klebsiella pneumoniae. J. Biotechnol. 168: 194-200.

34. Hasona A, Kim Y, Healy FG, Ingram LO, Shanmugam KT. 2004. Pyruvate formate lyase and acetate kinase are essential for anaerobic growth of Escherichia coli on xylose. J. Bacteriol. 186: 7593-7600. 
35. Lim JH, Seo SW, Kim SY, Jung GY. 2013. Model-driven rebalancing of the intracellular redox state for optimization of a heterologous n-butanol pathway in Escherichia coli. Metab. Eng. 20: 49-55

36. Zhang YP, Huang ZH, Du CY, Li Y, Cao ZA. 2009. Introduction of an NADH regeneration system into Klebsiella oxytoca leads to an enhanced oxidative and reductive metabolism of glycerol. Metab. Eng. 11: 101-106.

37. Jung MY, Mazumdar S, Shin SH, Yang KS, Lee J, Oh MK. 2014. Improvement of 2,3-butanediol yield in Klebsiella pneumoniae by deletion of the pyruvate formate-lyase gene. Appl. Environ. Microbiol. 80: 6195-6203.

38. Singh A, Lynch MD, Gill RT. 2009. Genes restoring redox balance in fermentation-deficient E. coli NZN111. Metab. Eng. 11: 347-354.

39. Zhu J, Shimizu K. 2004. The effect of pfl gene knockout on the metabolism for optically pure D-lactate production by Escherichia coli. Appl. Microbiol. Biotechnol. 64: 367-375.

40. Bar-Even A. 2016. Formate assimilation: the metabolic architecture of natural and synthetic pathways. Biochem. 55: $3851-3863$.

41. Chen T, Liu WX, Fu J, Zhang B, Tang YJ. 2013. Engineering Bacillus subtilis for acetoin production from glucose and xylose mixtures. J. Biotechnol. 168: 499-505.

42. Wang Y, Tao F, Xin B, Liu H, Gao Y, Zhou NY, et al. 2017. Switch of metabolic status: redirecting metabolic flux for acetoin production from glycerol by activating a silent glycerol catabolism pathway. Metab. Eng. 39: 90-101. 